\title{
Special Issue on CogInfoCom-Supported Approaches, Models and Solutions in Surface Transportation
}

Cognitive infocommunications (CogInfoCom) is an interdisciplinary research field that has emerged as a synergy between infocommunications and the cognitive sciences. One of the key observations behind CogInfoCom is that through a convergence process between these fields, humans and ICT are becoming entangled at various levels, as a result of which new forms of cognitive capability are appearing. Crucially, these capabilities are neither purely natural (i.e., human), nor purely artificial; therefore, it is suggested that they should be treated in a way that unifies both engineering and human-oriented perspectives, and based on concepts that underline the fact that a new form of co-evolutionary combo is replacing human-machine interactions.

This special issue focuses on applications of CogInfoCom relevant to surface transportation technologies. The goal is to provide an overview of areas that are often viewed as having little or no relationship to one another, but which at the same time can be approached under the same umbrella within CogInfoCom. Based on this common ground, the outlines become visible of new potential synergies between heretofore disparate disciplines.

The issue presents a collection of 7 papers focusing on vehicle-to-driver communication, vehicle-tovehicle communication, route planning/traffic control and high-level risk management. The paper by Ondas et al. and Sik et al. both focus on aspects relevant to the augmentation of communication between vehicles and humans - both at an individual and at a collec- tive level in the case of the latter paper. Problems relevant to inter-vehicle communication are addressed in the paper by Torok et al., which investigates whether driving mistakes can be detected earlier based on invehicle sensing systems than based on trajectory data. Challenges relevant to route planning and traffic control are addressed in papers by Winkler et al., Sylejmani et al. and Alfeo et al. Finally, high-level detection of potential safety risks is addressed in the paper by Mabrouk et al. using a hybrid system based on knowledge engineering and machine learning to detect potential hazards.

The recurrence of certain themes that are characteristically "cognitive" in the sense that they deal with human capabilities for sensing, reasoning and action - including themes such as cognitive load, socio-cognitive gamification, cognitive biases and so on - goes far to show how modeling human capabilities is increasingly important in engineering applications across the board. It is our hope that readers across a variety of disciplines will find something of interest in the special issue.

\section{The guest editors}

Peter Baranyi, Szechenyi Istvan University, Hungary

Attila Borsos, Szechenyi Istvan University, Hungary

Salvatore Cafiso, University of Catania, Italy

Marian Tracz, Cracow University of Technology, Poland 